\title{
Incidence, clinical features, and implications on outcomes of neonatal late-onset sepsis with concurrent infectious focus
}

I-Hsyuan Wu ${ }^{1,3}$, Ming-Horng Tsai ${ }^{1,2,3^{*}}$, Mei-Yin Lai ${ }^{1,3}$, Lee-Fen Hsu ${ }^{4}$, Ming-Chou Chiang ${ }^{1,3}$, Reyin Lien ${ }^{1,3}$, Ren-Huei Fu' ${ }^{1,3}$, Hsuan-Rong Huang ${ }^{1,3}$, Shih-Ming Chu ${ }^{1,3}$ and Jen-Fu Hsu ${ }^{1,3^{*}}$

\begin{abstract}
Background: Neonatal bloodstream infection (BSI) is the most important cause of morbidity and mortality in the neonatal intensive care unit (NICU). Although most neonatal BSIs are primary bacteremia, some are associated with a focus of infection. This distinction is not well characterized.

Methods: All patients with neonatal late-onset sepsis (LOS) between January 2006 and December 2013 were enrolled. LOS was categorized as a BSI with a concurrent focus of infection if LOS occurred before or within $24 \mathrm{~h}$ after the diagnosis of a specific infectious entity, and as "primary bacteremia" if no concurrent focus of infection was identified. Data concerning demographics, hospital course, microbiology, and outcomes were compared via univariate and multivariate analyses.
\end{abstract}

Results: Of 948 episodes of neonatal LOS, 781 (82.4\%) were primary bacteremia, whereas 167 (17.6\%) were associated with a known focus of infection, including meningitis $(n=51,5.4 \%)$, ventilator-associated pneumonia (VAP) ( $n=36,3.8 \%)$, catheter-related bloodstream infections ( $n=57,6.0 \%)$, and necrotizing enterocolitis (NEC) ( $n=21,2.2 \%$ ). The majority of NEC-associated BSIs were caused by gram-negative bacilli (85.7\%). Group B streptococcus accounted for nearly one-third of all meningitis cases (29.4\%). Although sepsis-attributable mortality was comparable between primary bacteremia and neonatal BSIs with a focus of infection, neonatal BSIs with meningitis, VAP, and NEC had significantly higher rates of infectious complications. The independent risk factors of sepsis-attributable mortality were infectious complications (Odds ratio [OR] 6.98; 95\% confidence interval [Cl] 3. 64-13.39, $P<0.001)$; history of one or more than one previous episode(s) of BSI (OR 2.40 and 7.40; 95\% Cl 1.21-4.74 and $3.70-14.78, P=0.012$ and $<0.001$, respectively); and underlying secondary pulmonary hypertension in neonates (OR 4.77; 95\% Cl 1.91-11.96, $P=0.001$ ).

Conclusions: A considerable proportion of neonatal LOS can be associated with known infectious foci in the NICU. The microbiologic etiology of neonatal LOS with a concurrent focus of infection is significantly different from that of primary bacteremia. Neonatal BSIs with concurrent meningitis, VAP, or NEC are significantly more likely to have infectious complications. This association independently leads to sepsis-attributable mortality.

Keywords: Bacteremia, Neonates, Catheter-related bloodstream infection, Late-onset sepsis, Risk factor

\footnotetext{
* Correspondence: mingmin.tw@yahoo.com.tw; jeff0724@gmail.com

${ }^{1}$ Division of Pediatric Neonatology, Department of Pediatrics, Chang Gung

Memorial Hospital, No. 5, Fu-Shin Rd., Kwei-Shan, Taoyuan, Linkou Chang

Gung Memorial Hospital, Taoyuan, Taiwan, Republic of China

Full list of author information is available at the end of the article
} 


\section{Background}

Bloodstream infection (BSI) is the most common nosocomial infection in the neonatal intensive care unit (NICU) $[1,2]$, and accounts for the most important cause of morbidity and mortality after these neonates have survived the perinatal insults and complications of extreme prematurity $[3,4]$. Neonates with certain underlying chronic comorbidities are more likely to have severe infection or die after BSI $[5,6]$. Factors predisposing to morbidity or mortality after neonatal BSI include prolonged use of a central catheter and/or ventilation, recurrent nosocomial infections, and possibly, immunosuppression caused by the use of broad-spectrum antibiotics [3-5]. Similarly, concurrent neonatal BSIs with infectious complications are often more severe, and sometimes may require surgical intervention $[7,8]$.

Our recent study has demonstrated that concurrent meningitis and ventilator-associated pneumonia were independent predictors of treatment failure in neonatal BSI [6]. Bizzarro et al. also showed that infants with necrotizing enterocolitis (NEC)-associated BSI are significantly more likely to die than are those with post-NEC BSI and NEC without BSI [9]. An episode of BSI with concurrent infectious focus may impose greater therapeutic challenge, and adequate infectious source control plays an important role in optimizing treatment response. There have been several studies in adults that investigated BSI with concurrent skin and soft tissue infection, bacteriuria, gastroenteritis, or pneumonia [1014], but the epidemiology and outcomes of neonatal BSIs with a concurrent infectious entity are poorly characterized. Therefore, we conducted a retrospective cohort study to determine the risk factors for, specific microbiology of, and clinical implications of neonatal BSI with a concurrent focus of infection.

\section{Methods}

\section{Study setting, subjects and ethics}

The Chang Gung Memorial Hospital (CGMH) is a tertiary-level medical center in a university-affiliated teaching hospital in northern Taiwan. The CGMH's NICU has three units that include a total of 49 beds equipped with mechanical ventilators, and 28 beds in special-care nurseries. All babies under 34-35 weeks gestation or birth weight $<2 \mathrm{~kg}$ or $>5 \mathrm{~kg}$, and those with any clinical signs of respiratory distress or cardiovascular, gastrointestinal, or neurological problems requiring surgical or intensive treatment were eligible for admission to this NICU. We identified all episodes of cultureproven BSI in the NICU of CGMH between 1 January 2006 and 31 December 2013. All BSIs identified were "late-onset", defined as a positive blood culture occurring at $>72$ h of life $[1,4,15]$. This study was approved by the institutional review board of Chang Gung
Memorial Hospital, with a waiver of informed consent because all patient records and information were anonymized and de-identified prior to analysis.

\section{Data retrieval and study database}

For more than ten years, the NICU at CGMH has had an electronic database maintained by a full-time nurse specialist dedicated to following neonates from birth (or admission if the neonate was transferred from another hospital) until discharge or death. The NICU database contains information on basic demographic data, perinatal insults, and complications of prematurity, a summary of the patient's hospital course, chronic comorbidities, and discharge diagnosis. Utilizing this database, we characterized in detail the course of every episode of BSI, including the clinical manifestations, laboratory results, microbiological data, concurrent focus of infection, treatment, infectious complications, and outcomes. The severity of illness was evaluated at the time of greatest severity during the course of BSI using the neonatal therapeutic intervention scoring system (NTISS) [16].

\section{Definitions}

An episode of BSI was defined according to the presence of clinical sepsis and the identification of pathogens, which included any bacteria isolated from at least one blood culture and not consisting of saprophytic skin flora [17]. Corynebacterium spp., Propionibacterium spp., Penicillium spp., and Diphtheroids spp. were considered contaminants in blood cultures, and were excluded from consideration. For coagulase-negative staphylococci (CoNS), the diagnosis of BSI required clinical signs of sepsis, and a blood culture positive for CoNS. The indicated treatment for CoNS BSI included intravenous antibacterial therapy for at least 5 days after the date of the blood culture (or until death) [1, 4]. We defined all concurrent infectious foci, including NEC, ventilator associated pneumonia (VAP), central lineassociated bloodstream infection (CLABSI), and meningitis according to the U.S. Centers for Disease Control and Prevention's (CDC's) National Healthcare Safety Network (NHSN) definitions [8, 18-20]. Only cases with NEC $\geq$ stage IIA in modified Bell's criteria (definite NEC) were enrolled, and clinically defined VAP cases were considered [8]. An episode of BSI was defined as "BSI with concurrent infectious focus" if the onset of BSI (defined as the timing of the first positive blood culture was drawn) was accompanied with a specific infectious entity, i.e. meningitis, VAP, or NEC, otherwise, this BSI was categorized as "primary bacteremia". In this definition, the onset of BSI was always within $24 \mathrm{~h}$ (before or after) of the diagnosis of a specific infectious entity.

A patient was said to have an episode of clinical sepsis if the patient had a positive blood culture and was 
treated with antibiotic therapy for 5 or more days (or was treated for a shorter period if the patient died) and had at least two of the following clinical symptoms of sepsis: fever or hypothermia, hyper- or hypoglycemia, apnea or tachypnea, frequent oxygen desaturation with an increased requirement for ventilator support, bradycardia and/or cyanosis, feeding intolerance, abdominal distension, seizures, decreased motor activity, skin mottling, and hypotension [1, 4]. The microbiological assay system in our hospital is a matrix-assisted laser desorption ionization time-of-flight (MALDI-TOF) system (Bruker's flagship FLEX series) [21].

The diagnosis of all co-morbidities of prematurity, including respiratory distress syndrome (RDS), intraventricular hemorrhage (IVH), broncho-pulmonary dysplasia (BPD), necrotizing enterocolitis (NEC), and peri-ventricular leukomalacia (PVL) was based on the latest updated diagnostic criteria [22]. Congenital anomalies included either documented or undocumented syndromes, chromosomal abnormalities, and genetic or metabolic disorders, but not simple cleft palate or polydactyly. Persistent BSI or fungemia was defined as three or more consecutive positive blood cultures, at least $48 \mathrm{~h}$ apart, during a single episode of sepsis [23].

Infectious complications were defined as a new-onset focus of infection, empyema, abscess, venous thrombosis, or vegetation directly related to bacteremia or major organ dysfunction within 1 week after the onset of bacteremia. Empiric antibiotic therapy was considered inappropriate if the treatment regimen did not include at least one antibiotic active in vitro against the infecting microorganisms, administered within $24 \mathrm{~h}$ of blood culture collection. For patients who died during hospitalization, the cause of death was recorded according to the clinician's assessment. Sepsis-attributable mortality was defined as death of a neonate within 3 days after the onset of sepsis or death from of infectious complications or clinically progressive deterioration following the onset of BSI.

\section{Statistical analysis}

Descriptive data all episodes of neonatal BSI were expressed as mean and standard deviation (SD) or median and IQR (interquartile range), where appropriate, for continuous data and absolute number and percentage for dichotomous data. Infecting organisms responsible for BSI in infants with infectious entities associated and primary BSI were compared via $X^{2}$ analysis and presented as unadjusted OR with $95 \%$ CI. If an infant had multiple episodes of sepsis, each episode was considered as an independent event.

In univariate analyses, continuous data were analyzed using the Kruskal-Wallis and Mann-Whitney U tests, where appropriate. Dichotomous data were analyzed using $X^{2}$ test and Fisher exact test, where appropriate. A $P$ value $<0.05$ was considered statistically significant. BSI-attributable mortality was assessed as the dependent variable in a multivariate logistic regression model. This model incorporated variables identified in a univariate regression model with a $P$ value of $<0.10$. Estimates were expressed as odds ratios (ORs) with 95\% confidence intervals (CIs). All statistical analyses were performed using SPSS version 21.0 (SPSS, Chicago, IL, USA).

\section{Results}

A total of 948 episodes of BSI were identified in 732 infants during this study period, of which 781 (82.4\%) were primary bacteremia and $167(17.6 \%)$ had a specific focus of infection. The incidence rate of neonatal BSI during this study period was 3.71 per 1000 neonate-hospital days. Among those with concurrent infection, 51 (45\%) BSIs had meningitis, 36 (23\%) were VAP, 21 (23\%) were NEC, and 57 (28\%) were CLABSI. Other uncommon foci of infection included osteomyelitis (2), septic arthritis (2), upper limbs cellulitis (1), and urinary tract infection (1). Among these 167 BSIs with a focus of infection, five $(3.0 \%)$ had more than two infectious foci. The microbiology of BSIs with concurrent infection was distinctively different from that of primary bacteremia (Table 1). The majority of NEC-associated BSIs were caused by gram-negative bacilli (85.7\%) and group B streptococcus accounted for nearly one-third of all meningitis cases (29.4\%). More than half cases of VAPand CLABSI-associated BSIs were attributable to gramnegative bacilli (61.1\%) and gram-positive pathogens (64.9\%), respectively.

Demography, hospital course, underlying chronic comorbidities, and treatment for all neonatal BSIs with concurrent infection were evaluated comparing with primary BSIs (Table 2). Neonates with BSIs concurrent with meningitis had a significantly higher birth weight and later gestational age than neonates with primary bacteremia, whereas neonates with BSIs concurrent with VAP had a significantly lower birth weight and gestational age than neonates with primary bacteremia. Neonatal BSIs with concurrent NEC and neonatal BSIs with concurrent VAP tended to occur earlier and later than primary bacteremia, respectively. VAP associated BSIs were more likely to occur in neonates with low Apgar score $(\leq 7)$ at five minutes and in those with perinatal asphyxia.

The underlying chronic comorbidities were not significantly different between primary bacteremia and BSIs with a concurrent focus of infection, except that VAPassociated BSIs and meningitis were more likely to occur in neonates with BPD and neurological comorbidities, respectively. In addition to GBS meningitis, other grampositive-cocci-associated meningitis occurred mostly in neonates with congenital or acquired hydrocephalus as a 
Table 1 Pathogens causing a total of 948 episodes of primary bloodstream infection (BSI) and 123 episodes of BSI with specific infectious focus in the neonatal intensive care unit

\begin{tabular}{|c|c|c|c|c|c|c|}
\hline & \multicolumn{5}{|c|}{ Neonatal BSIs with specific infectious focus (total $n=167$ ) } & \multirow{2}{*}{$\begin{array}{l}\text { Primary bacteramia } \\
\text { (total } n=781 \text { ) }\end{array}$} \\
\hline & $\begin{array}{l}\text { Meningitis } \\
\text { (total } n=51 \text { ) }\end{array}$ & $\begin{array}{l}\text { Ventilator associated } \\
\text { pneumonia }(n=36)\end{array}$ & $\begin{array}{l}\text { CLABSI } \\
(n=57)\end{array}$ & $\begin{array}{l}\text { Necrotizing enterocolitis } \\
(n=21)\end{array}$ & $\begin{array}{l}\text { Others }^{c} \\
(n=7)\end{array}$ & \\
\hline \multicolumn{7}{|l|}{ Gram-positive organism } \\
\hline Coagulase-negative Staphylococcus & $4(7.8)$ & $0(0)$ & $25(43.9)$ & $3(14.3)$ & $1(14.3)$ & $332(42.5)$ \\
\hline Staphylococcus aureus & $9(17.6)$ & $9(25.0)$ & $11(19.3)$ & $0(0)$ & $4(57.1)$ & $89(11.4)$ \\
\hline Enterococcus species & $2(3.9)$ & $1(2.8)$ & $1(1.8)$ & $0(0)$ & $0(0)$ & $22(2.8)$ \\
\hline Group-B streptococcus & $15(29.4)$ & $1(2.8)$ & $0(0)$ & $0(0)$ & $0(0)$ & $10(1.3)$ \\
\hline Streptococcus pneumoniae & $0(0)$ & $1(2.8)$ & $0(0)$ & $0(0)$ & $0(0)$ & $2(0.2)$ \\
\hline Viridan Streptococcus & $0(0)$ & $0(0)$ & $0(0)$ & $0(0)$ & $0(0)$ & $2(0.2)$ \\
\hline \multicolumn{7}{|l|}{ Gram-negative organism } \\
\hline Klebsiella pneumoniae & $5(9.8)$ & $6(16.7)$ & $5(8.8)$ & $3(14.3)$ & $0(0)$ & $72(9.2)$ \\
\hline Klebsiella oxytoca & $0(0)$ & $1(2.8)$ & $1(1.8)$ & $0(0)$ & $0(0)$ & $22(2.8)$ \\
\hline Escherichia coli & $7(13.7)$ & $3(8.3)$ & $2(3.5)$ & $8(38.1)$ & $1(14.3)$ & $59(7.5)$ \\
\hline Enterobacter cloacae & $1(2.0)$ & $1(2.8)$ & $1(1.8)$ & $0(0)$ & $0(0)$ & $21(2.7)$ \\
\hline Enterobacter aerogenes & $0(0)$ & $1(2.8)$ & $1(1.8)$ & $1(4.8)$ & $0(0)$ & $13(1.7)$ \\
\hline Pseudomonas aeruginosa & $2(3.9)$ & $2(5.6)$ & $1(1.8)$ & $1(4.8)$ & $0(0)$ & $10(1.3)$ \\
\hline Acinetobacter baumannii & $1(2.0)$ & $3(8.3)$ & $1(1.8)$ & $1(4.8)$ & $0(0)$ & $36(4.6)$ \\
\hline Serratia marcescens & $2(3.9)$ & $1(2.8)$ & $2(3.5)$ & $2(9.5)$ & $0(0)$ & $6(0.8)$ \\
\hline Others $^{a}$ & $2(3.9)$ & $1(2.8)$ & $1(1.8)$ & $0(0)$ & $0(0)$ & $8(1.3)$ \\
\hline \multicolumn{7}{|l|}{ Fungus } \\
\hline Candida albicans & $1(2.0)$ & $0(0)$ & $4(7.0)$ & $2(5.6)$ & $0(0)$ & $18(2.3)$ \\
\hline Candida parapsilosis & $0(0)$ & $0(0)$ & $1(1.8)$ & $0(0)$ & $0(0)$ & $13(1.7)$ \\
\hline Other Candida spp. & $0(0)$ & $0(0)$ & $0(0)$ & $0(0)$ & $0(0)$ & $11(1.4)$ \\
\hline Polymicrobial microorganisms ${ }^{b}$ & $0(0)$ & $3(8.3)$ & $0(0)$ & $2(9.5)$ & $1(14.3)$ & $35(4.5)$ \\
\hline
\end{tabular}

CLABSI central line-associated bloodstream infection

ancluding Citrobacter freundii (3), Stenotrophomonas maltophilia (3), Hafnia alvei (2), Neisseria meningitidis (2), Chryseobacterium meningoseptium (1) and Flavobacterium (1)

Indicating two or more microorganisms were recovered from the same blood culture set

Including osteomyelitis (2), septic arthritis (2), upper limbs cellulitis (2), and urinary tract infection (1)

complication of ventriculoperitonal shunt or extraventricular drainage. All episodes of primary bacteremia, as well as neonatal BSIs with concurrent meningitis, VAP, or CLABSI were evenly distributed as first episode of LOS or recurrent episode of LOS; however, there were only two cases of BSI with concurrent NEC occurring as a recurrent episode of LOS.

The clinical presentations of neonatal BSI with meningitis and CLABSIs were mostly comparable to those of primary bacteremia; however, NEC-associated and VAPassociated BSIs had more severe clinical symptoms, including more septic shock, disseminated intravascular coagulopathy, thrombocytopenia, anemia, and metabolic acidosis. Appropriate antibiotics were administrated in $72.2 \%$ of cases of primary bacteremia within $24 \mathrm{~h}$ of onset, which was similar to the rate in cases of neonatal BSIs with concurrent VAP, NEC, and CLABSIs.

Neonatal BSIs with concurrent VAP and NEC had significantly greater severity of illness, judged by NTISS scores (Table 3). Neonates with VAP-associated BSIs were more likely to require prolonged invasive intubation ( $\geq 7$ days) and to experience respiratory failure under a high-frequency oscillatory ventilator. Neonatal BSIs with concurrent meningitis, VAP, and NEC had significantly higher rates of infectious complications than did primary bacteremia. The sepsis-attributable mortality rates were comparable between primary bacteremia and BSIs with a concurrent focus of infection.

Table 4 shows univariate comparisons of neonatal BSIs with sepsis-attributable mortality versus those without. None of neonatal BSIs with concurrent meningitis, VAP, or NEC was associated with significantly higher rates of sepsis-attributable mortality. Underlying secondary pulmonary hypertension (due to severe BPD), neurological comorbidities, presence of infectious complications, and history of one or more previous episode(s) of neonatal BSI were significantly associated with a higher risk of sepsisattributable mortality. In multivariate logistic regression, 


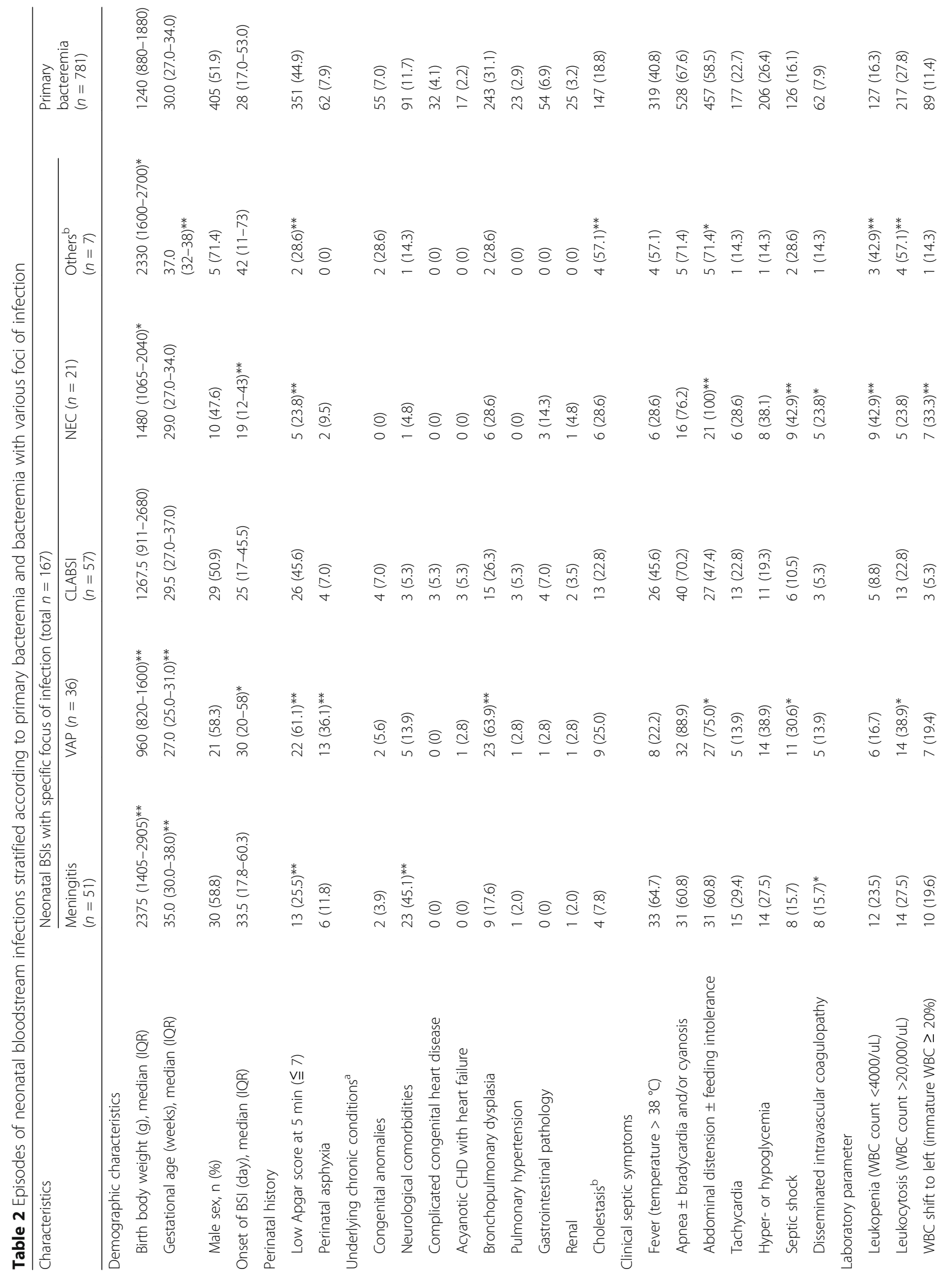




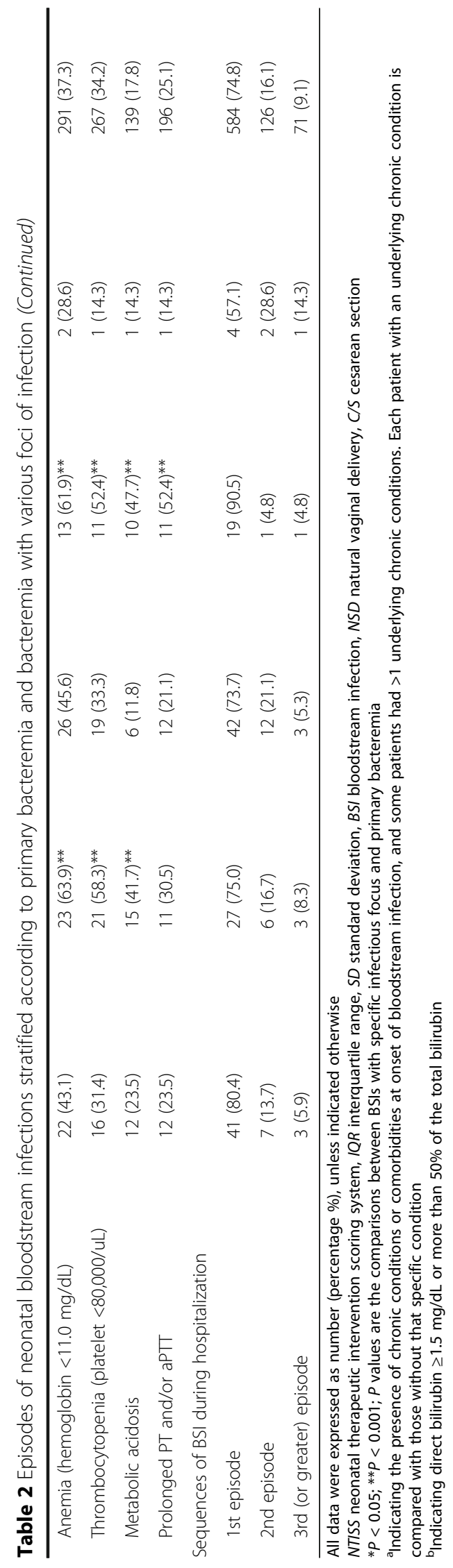


Table 3 Treatment and outcomes of neonatal bloodstream infections among the three CRP groups

\begin{tabular}{|c|c|c|c|c|c|c|}
\hline & \multicolumn{5}{|c|}{ Neonatal BSIs with specific infectious focus (total $n=167$ ) } & \multirow{2}{*}{$\begin{array}{l}\text { Primary bacteremia } \\
(n=781)\end{array}$} \\
\hline & $\begin{array}{l}\text { Meningitis } \\
(n=51)\end{array}$ & $\begin{array}{l}\text { VAP } \\
(n=36)\end{array}$ & $\begin{array}{l}\text { CLABSI } \\
(n=57)\end{array}$ & $\begin{array}{l}\text { NEC } \\
(n=21)\end{array}$ & $\begin{array}{l}\text { Others }{ }^{\#} \\
(n=7)\end{array}$ & \\
\hline NTISS score, (mean \pm SD) & $15.4 \pm 5.1$ & $19.8 \pm 4.0^{* *}$ & $16.8 \pm 4.0$ & $19.0 \pm 6.5^{* *}$ & $14.7 \pm 6.1$ & $16.9 \pm 4.5$ \\
\hline \multicolumn{7}{|l|}{ Ventilator requirement } \\
\hline Prolonged invasive intubation ( $\geq 7$ days) & $14(27.5)$ & $22(61.1)^{* *}$ & 19 (33.3) & $8(38.0)$ & $2(28.6)$ & $287(36.7)$ \\
\hline High frequency oscillatory ventilator & $0(0)$ & $14(38.9)^{* *}$ & $3(5.3)$ & $4(19.0)^{*}$ & $0(0)$ & $71(9.1)$ \\
\hline Inadequate antibiotic treatment within the first $24 \mathrm{~h}$ & $10(19.6)^{*}$ & $8(22.2)$ & $17(29.8)$ & $7(33.3)$ & $4(57.1)^{* *}$ & $217(27.8)$ \\
\hline \multicolumn{7}{|l|}{ Outcomes } \\
\hline Infectious complications & $18(35.3)^{* *}$ & $10(27.8)^{* *}$ & $6(10.5)$ & $5(23.8)^{* *}$ & $0(0)$ & $68(8.7)$ \\
\hline Sepsis attributable mortality & $5(9.8)$ & $4(11.1)$ & $3(5.3)$ & $3(14.3)$ & $1(14.3)$ & $53(6.8)$ \\
\hline
\end{tabular}

The $P$ values are the comparisons between neonatal BSIs with infectious focus and primary bacteremia

*P $P$ < 0.05

${ }^{* *} P<0.001$

the presence of infectious complications (Odds ratio [OR] 6.98; 95\% confidence interval $[\mathrm{CI}] 3.64-13.39, P<0.001$ ), history of one or more previous episode(s) of BSI (OR 2.40 and $7.40 ; 95 \%$ CI $1.21-4.74$ and $3.70-14.78$, $P=0.012$ and $<0.001$, respectively), and underlying secondary pulmonary hypertension (OR 4.77; 95\% CI $1.91-11.96, P=0.001$ ) were independent predictors of sepsis-attributable mortality.

\section{Discussion}

In this study, we found that the majority of neonatal late-onset BSIs were primary bacteremia, and only $17.6 \%$ were associated with a specific focus of infection. To our knowledge, this is the first investigation of neonatal BSIs with concurrent onset of nosocomial infections. Not surprisingly, the microbiologic profile of neonatal BSI concurrent with a specific focus of infection was significantly different from that of primary bacteremia. In contrast to previous studies documenting that patients with NEC-associated BSI and GBS meningitis have higher mortality rates than do those with bacteremia $[9$, 24], we found that a concurrent focus of infection did not directly contribute to case mortality. Instead, a concurrent focus of infection was associated with a significantly higher rate of infectious complications, and the associations finally lead to sepsis attributable mortality.

The overall incidence of culture-positive meningitis, VAP, NEC, and the distribution of organisms in this

Table 4 Risk factors for sepsis-attributable mortality in neonatal bloodstream infections with and without a focus of infection

\begin{tabular}{|c|c|c|c|c|}
\hline \multirow[t]{2}{*}{ Variables } & \multicolumn{2}{|l|}{ Univariate analysis } & \multicolumn{2}{|c|}{ Multivariate logistic regression analysis } \\
\hline & Unadjusted OR (95\% Cl) & $P$ value & Adjusted OR (95\% Cl) & $P$ value \\
\hline Gestational age (weeks) & $0.95(0.90-1.01)$ & 0.126 & - & - \\
\hline Inappropriate antibiotics & $1.64(1.01-2.73)$ & 0.048 & $1.05(0.57-1.94)$ & 0.872 \\
\hline Presence of infectious complications & $6.19(3.61-10.62)$ & $<0.001$ & $6.98(3.64-13.39)$ & $<0.001$ \\
\hline \multicolumn{5}{|l|}{ Sequence of bloodstream infections } \\
\hline 1st episode & 1 (reference) & & 1 (reference) & \\
\hline 2nd episode & $2.90(1.57-5.41)$ & 0.001 & $2.40(1.21-4.74)$ & 0.012 \\
\hline 3rd (or greater) episode & $8.53(4.63-15.71)$ & $<0.001$ & $7.40(3.70-14.78)$ & $<0.001$ \\
\hline Underlying pulmonary hypertension & $8.11(3.58-18.35)$ & $<0.001$ & $4.77(1.91-11.96)$ & 0.001 \\
\hline Underlying neurological comorbidities & $2.80(1.59-4.93)$ & $<0.001$ & $1.89(0.99-3.61)$ & 0.053 \\
\hline \multicolumn{5}{|l|}{ BSIs with and without infectious focus } \\
\hline Primary bacteremia & 1 (reference) & & - & - \\
\hline With concurrent meningitis & $1.05(0.34-3.21)$ & 0.937 & - & - \\
\hline With concurrent ventilator-associated pneumonia & $1.27(0.38-4.20)$ & 0.699 & - & - \\
\hline With necrotizing enterocolitis & $2.84(0.64-12.57)$ & 0.169 & - & - \\
\hline With concurrent CLABSIs & $1.07(0.34-3.75)$ & 0.918 & - & - \\
\hline
\end{tabular}


cohort was similar to other studies [9, 24-26]. Although neonates with concurrent VAP and NEC seemed more severely ill, the insignificant influence of neonatal meningitis, VAP, and NEC on sepsis-attributable mortality can be explained partially by the small sample size. Our previous studies showed that neonates with fungal BSI and multidrug-resistant gram-negative bacteremia have a significantly higher mortality rate $[1,5]$. Because these pathogens usually caused primary bacteremia in this study, the sepsis-attributable mortality was comparable between primary bacteremia and bacteremia with concurrent infectious focus. Furthermore, our aggressive treatment strategies for neonatal NEC, as well as meningitis and VAP, could possibly reduce the mortality rate of these disease entities $[1,2,5,6]$.

Although our study extended over a relatively long period of time, there were very few variations in practice and all collections of data showed similar results (data not shown). The sepsis-attributable mortality rates in our cohort, both in the primary bacteremia group and in the group with BSI with a concurrent focus of infection, were much lower than those reported in other studies $[9,27]$. For neonatal BSIs with meningitis, the case fatality rate of our cohort was comparable to that reported in some recent studies $[24,28$, 29]. Previous studies have shown worse outcomes in preterm or small for gestational age infants [28-30], but this was not documented in our study. It is therefore likely that the presence of underlying chronic comorbidities contributed to mortality $[1,6]$, both in primary bacteremia and neonatal BSIs with a concurrent focus of infection.

A recent study found that infants with bloodcerebrospinal fluid (CSF) concordance were more likely to have indicators related to increased severity of illness [26]. Because neonatal meningitis is inevitably associated with significantly higher rates of mortality and morbidity $[6,7$, 31 , the relatively lower sepsis-attributable mortality of neonatal BSI with meningitis in this cohort highlighted the importance of aggressive treatment. Based on this result, the clinicians should consider treating the primary bacteremia and controlling the infectious focus at the same time once a focus is identified. The antibiotic regimens and treatment duration of neonatal bacteremia with concurrent meningitis or NEC are supposed to be adjusted to the presence of infectious complications.

CLABSI is an important source of neonatal BSI. In this study, we applied strict diagnostic criteria for CLABSI [18]. CLABSI was diagnosed only in neonates from whom the catheter was removed at the time of or soon after the onset of the episode of BSI, and in whom culture of the catheter tip was positive for the same organism that was cultured from the blood. Cultures are imperfect, and sometimes the catheter was removed after empiric antibiotics had been administrated. Therefore, we may have inadvertently missed patients who, in fact, had an unidentified focus of infection in the catheter. Furthermore, some CLABSIs were treated with the catheter in situ, which led to an underestimate of the true incidence of neonatal BSI due to CLABSIs. Nonetheless, it is clear that in almost all demographics, clinical characteristics, treatment, and outcomes neonatal BSIs due to CLABSIs were comparable to those details in primary bacteremia.

The presence of certain underlying chronic comorbidities may increase the risk of secondary bacteremia. In our cohort, only neurological comorbidities and BPD predisposed neonates to higher rates of meningitis and VAP, respectively. The frequency of secondary bacteremia in neonates with nosocomial infections other than BSIs was low $[32,33]$, but it should be considered when there are clinical symptoms lasting more than $48 \mathrm{~h}$ or when signs or symptoms of sepsis have reemerged.

The sources of neonatal BSIs were identified in only $17.6 \%$ episodes in this cohort. It has been proven that the sources of neonatal BSIs are most commonly from epidermis colonization and gram-negative bacilli penetration of bowel mucosa, for gram-positive cocci and gram-negative bacilli, respectively $[34,35]$. Because not all infected catheters were removed for bacterial culture at onset of BSIs, the percentage of neonatal BSIs with concurrent CLABSI in this cohort may be underestimated.

The microbiological assay system is MALDI-TOF in our institute, which has the advantage of rapid identification and reliable microbiological results [36, 37]. However, the disadvantage of this method is the lacking tests of antibiotic resistances; therefore, additional antibiotic susceptibility testing was required in our institute.

This study has some limitations. This was a retrospective, single-center cohort study, which inevitably restricts its generalizability compared with that of a prospective, multicenter study. We applied the definition of single positive blood culture for CoNS with clinical symptoms, which possibly resulted in an over-estimate of the burden of CoNS-related bacteremia. Subgroup analyses were limited by sample size, and some episodes of BSIs with a focus of infection may have been treated with antibiotics while awaiting blood culture results. The delay in identifying a specific focus may have resulted in sterilization of the CSF, sputum, or catheter tips. Blood cultures were ordered as clinically indicated, and the pathogens might not have been identified in some patients with infectious sources. Further, the identification of concurrent infectious was based on clinical judgment, which can be subjective.

\section{Conclusions}

Our data suggest that neonatal BSIs with a concurrent focus of infection is not rare, and is associated with increased severity of illness and a higher rate of infectious complications. At the initial presentation of neonatal 
BSI, systemic evaluation should be performed to identify a possible source of infection after blood cultures are obtained. In the support and recovery phase of neonatal BSIs with a concurrent focus of infection, especially in cases with VAP, meningitis, and NEC, efforts to limit the risk of infectious complications and prolonged hospitalization are necessary, because infectious complications are independently associated with in-hospital mortality.

\begin{abstract}
Abbreviations
BPD: Bronchopulmonary dysplasia; BSI: Bloodstream infection; CDC: Centers for disease control and prevention; Cl: Confidence interval; CoNS: Coagulase negative staphylococcus; CRBSI: Catheter-related bloodstream infection; CRP: C-reactive protein; CVC: Central venous catheter; ESBL: Extendedspectrum $\beta$-lactamase; GBS: Group B streptococcus; GPC: Gram-positive cocci; IQR: Interquartile range; MALDI-TOF: Matrix-assisted laser desorption ionization time-of-flight; NEC: Necrotizing enterocolitis; NICU: Neonatal intensive care unit; NTISS: Neonatal therapeutic intervention scoring system; OR: Odds ratio; PVL: Periventricular leukomalacia; RDS: Respiratory distress syndrome; TPN: Total parenteral nutrition; VAP: Ventilator associated pneumonia
\end{abstract}

\section{Acknowledgements}

The authors thank Mrs. Chiao-Ching Chiang for keeping the database of our $\mathrm{NICU}$, and all nursing staff working in our NICUs for keeping extremely detailed patient records, which contributed greatly to the completion of this research. We want to thank Miss Chun-Chun Cheng and Mr. Yu-Jr. Lin for statistical consultation, supported by grants from Biostatistical Center for Clinical Research, Chang Gung Memorial Hospital (CLRPG340599).

\section{Availability of data and materials}

The manuscript detailing where the data supporting the findings in this study can be found if requested.

\section{Authors' contributions}

I-HW conceptualized and designed the study, drafted the initial manuscript, and approved the final manuscript as submitted. M-HT took care of patients in the study, collected and verified the data, and assisted in drafting the manuscript. M-YL and L-FH designed the data collection instruments, coordinated and supervised data collection, and assisted in drafting the manuscript. M-CC took care of patients in the study, helped with data verification, and assisted in drafting the manuscript. RL took care of patients in the study, approved the agreement with the institutional review board, and assisted in drafting the manuscript. R-HF took care of patients in the study, helped with data verification, and assisted in drafting the manuscript $\mathrm{H}-\mathrm{RH}$ took care of patients in the study, carried out the initial analyses, and assisted in drafting the manuscript. S-MC took care of patients in the study, collected and verified data, critically reviewed the manuscript, revised the manuscript, and approved the final manuscript as submitted. J-FH critically reviewed the manuscript, revised the manuscript, and approved the final manuscript as submitted. All authors have read and approve of the final version of the manuscript.

\section{Ethics approval and consent to participate}

This study was approved by the institutional review board of Chang Gung Memorial Hospital, with a waiver of informed consent because all patient records and information were anonymized and de-identified prior to analysis.

\section{Consent for publication}

Not applicable.

\section{Competing interests}

The authors declare that they have no competing interests.

\section{Publisher's Note}

Springer Nature remains neutral with regard to jurisdictional claims in published maps and institutional affiliations.

\section{Author details}

'Division of Pediatric Neonatology, Department of Pediatrics, Chang Gung Memorial Hospital, No. 5, Fu-Shin Rd., Kwei-Shan, Taoyuan, Linkou Chang Gung Memorial Hospital, Taoyuan, Taiwan, Republic of China. ${ }^{2}$ Division of Neonatology and Pediatric Hematology/Oncology, Department of Pediatrics, Chang Gung Memorial Hospital, Yunlin, Taiwan. ${ }^{3}$ College of Medicine, Chang Gung University, Taoyuan, Taiwan. ${ }^{4}$ Department of Respiratory Care, Chang Gung University of Science and Technology, Chia-Yi, Taiwan.

Received: 27 September 2016 Accepted: 29 June 2017

Published online: 03 July 2017

\section{References}

1. Tsai MH, Hsu JF, Chu SM, Lien R, Huang HR, Chiang MC, et al. Incidence, clinical characteristics, and risk factors of adverse outcome in neonates with late-onset sepsis. Pediatr Infect Dis J. 2014;33:e7-e13.

2. Tsai MH, Wu IH, Lee CW, Chu SM, Lien R, Huang HR, et al. Neonatal gramnegative bacillary late-onset sepsis: a case-control-control study on a prospectively collected database of 5233 admissions. Am J Infect Control. 2016:44:146-53.

3. Tsai MH, Lien R, Wang JW, Huang HR, Chiang CC, Chu SM, et al. Complication rates with percutaneously central venous catheters inserted at femoral and non-femoral sites in very low birth weight infants. Pediatr Infect Dis J. 2009;28:966-70.

4. Tsai MH, Chu SM, Lee CW, Hsu JF, Huang HR, Chiang MC, et al. Recurrent late-onset sepsis in the neonatal intensive care unit: incidence, clinical characteristics, and risk factors. Clin Microbiol Infect. 2014;20:0928-35.

5. Tsai MH, Chu SM, Hsu JF, Lien R, Huang HR, Chiang MC, et al. Risk factors and outcomes for multidrug-resistant gram-negative bacteremia in the NICU. Pediatrics. 2014;133:e322-9.

6. Hsu JF, Chu SM, Huang YC, Lien R, Huang HR, Lee CW, Chiang MC, Fu RH, Tsai $\mathrm{MH}$. Predictors of clinical and microbiological treatment failure in neonatal bloodstream infections. Clin Microbiol Infect 2015;21:e482.e9-482.e17.

7. Chu SM, Hsu JF, Lee CW, Lien R, Huang HR, Chiang MC, Fu RH, Tsai MH. Neurological complications after neonatal bacteremia: the clinical characteristics, risk factors, and outcomes. PLoS One. 2014;9:e105294.

8. Chu SM, Yang MC, Hsiao HF, Hsu JF, Lien R, Chiang MC, Fu RH, Huang HR, Hsu $\mathrm{KH}$, Tsai MH. One-week versus 2-day ventilator circuit change in neonates with prolonged ventilation: cost-effectiveness and impact on ventilator-associated pneumonia. Infect Control Hosp Epidemiol. 2015;36:287-93.

9. Bizzarro MJ, Ehrenkranz RA, Gallagher PG. Concurrent bloodstream infection in infants with necrotizing enterocolitis. J Pediatr. 2014;164:61-6.

10. Tattevin P, Schwartz BS, Graber CJ, Volinski J, Bhukhen A, Mai TT, et al. Concurrent epidemics of skin and soft tissue infection and bloodstream infection due to community-associated methicillin-resistant Staphylococcus Aureus. Clin Infect Dis. 2012;55:781-8.

11. Yoon YK, Lee J, Ryu SY, Chang HH, Choi WS, Yoon JH, et al. Clinical significance of multidrug-resistant Acinetobacter Baumannii isolated from central venous catheter tip cultures in patients without concomitant bacteremia. Scand J Infect Dis. 2013;45:900-6.

12. Schreiber MP, Chan CM, Shorr AF. Bacteremia in Staphylococcus Aureus pneumonia: outcomes and epidemiology. J Crit Care. 2011;26:395-401.

13. Choi SH, Lee SO, Choi JP, Lim SK, Chung JW, Choi SH, et al. The clinical significance of concurrent Staphylococcus Aureus bacteriuria in patients with S. Aureus bacteremia. J Inf Secur. 2009:59:37-41.

14. Puerta-Fernandez S, Miralles-Linares F, Sanchez-Simonet MV, Bernal-Lopez MR, Gomez-Huelgas R. Raoultella planticola bacteraemia secondary to gastroenteritis. Clin Microbiol Infect. 2013;19:E236-7.

15. Verstraete EH, Blot K, Mahieu L, Vogelaers D, Blot S. Prediction models for neonatal health care-associated sepsis: a meta-analysis. Pediatrics. 2015;135:e1002-14.

16. Gray JE, Richardson DK, McCormick MC, Workman-Daniels K, Goldmann DA. Neonatal therapeutic intervention scoring system: a therapy-based severityof-illness index. Pediatrics. 1992;90:561-7.

17. Opota O, Croxatto A, Prod'hom G, Greub G. Blood culture-based diagnosis of bacteraemia: state of the art. Clin Microbiol Infect. 2015;21:313-22.

18. Mehta Y, Jaggi N, Rosenthal VD, Kavathekar M, Sakle A, Munshi N, et al. Device-associated infection rates in 20 cites of India, data summary for 2004-2014: findings of the international nosocomial infection control consortium. Infect Control Hosp Epidemiol. 2016;37:172-81. 
19. Tsai MH, Chu SM, Hsu JF, Lien R, Huang HR, Chiang MC, et al. Breakthrough bacteremia in the neonatal intensive care unit: incidence, risk factors, and attributable mortality. Am J Infect Control. 2015;43:20-5.

20. Al-Mousa HH, Omar AA, Rosenthal VD, Salama MF, Aly NY, El-Dossoky Noweir M, et al. Device-associated infection rates, bacterial resistance, length of stay, and mortality in Kuwait: international nosocomial infection consortium findings. Am J Infect Control. 2016;44:444-9.

21. Rodriguez-Sánchez B, Sánchez-Carrillo C, Ruiz A, Marin M, Cercenado E, Rodrigez-Créixems M, Bouza E. Direct identification of pathogens from positive blood cultures using matrix-assisted laser desorption-ionization time-of-flight mass spectrometry. Clin Microbiol Infect. 2014;20:0421-7.

22. Gleason CA, Devaskar SU. Avery's disease of the newborn. 9th ed. Philadelphia, PA: Elsevier Saunders; 2011.

23. Hsu JF, Chu SM, Lee CW, Yang PH, Lien R, Chiang MC, et al. Incidence, clinical characteristics and attributable mortality of persistent bloodstream infection in the neonatal intensive care unit. PLoS One. 2015;10:e0124567.

24. Joubrel C, Tazi A, Six A, Dmytruk N, Touak G, Bidet P, et al. Group B streptococcus neonatal invasive infection, France 2007-2012. Clin Microbiol Infect. 2015;21:910-6.

25. Stoll BJ, Hansen NI, Bell EF, Shankaran S, Laptook AR, Walsh MC, et al. Neonatal outcomes of extremely preterm infants from the NICHD neonatal research network. Pediatrics. 2010;126:443-56.

26. Beam KS, Laughon MM, Hornik CP, Cohen-Wolkowiez M, Clark RH, Benjamin DK Jr, et al. Predictors of positive cerebrospinal fluid cultures in infants with bacteremia. Pediatr Infect Dis J. 2014;33:360-5.

27. Schwab F, Zibell R, Piening B, Geffers C, Gastmeier P. Mortality due to bloodstream infections and necrotizing enterocolitis in very low birth weight infants. Pediatr Infect Dis J. 2015;34:235-40.

28. Okike IO, Johnson AP, Henderson KL, Blackburn RM, Muller-Pebody B, Ladhani SN, et al. Incidence, etiology, and outcome of bacterial meningitis in infants aged < 90 days in the United Kingdom and Republic of Ireland: prospective, enhanced, national population-based surveillance. Clin Infect Dis. 2014:59:e150-7.

29. Gaschignard J, Levy C, Romain O, Cohen R, Bingen E, Aujard Y, Boileau P. Neonatal bacterial meningitis: 444 cases in 7 years. Pediatr Infect Dis J. 2011;30:212-7

30. Escobar GJ, Puopolo KM, Wi S, Turk BJ, Kuzniewicz MW, Walsh EM, et al. Stratification of risk of early-onset sepsis in newborn $\geqq 34$ weeks' gestation. Pediatrics. 2014;133:30-6.

31. Shane AL, Hansen NI, Stoll BJ, Bell EF, Sánchez PJ, Shankaran S, et al. Methicillin-resistant and susceptible Staphylococcus Aureus bacteremia and meningitis in preterm infants. Pediatrics. 2012;129:e914-22.

32. Gözmen S, Sükran Gözmen K, Apa H, Aktürk H, Sorguç Y, Bayram N, et al. Secondary bacteremia in rotavirus gastroenteritis. Pediatr Infect Dis J. 2014;33:775-7

33. Lowenthal A, Livni G, Amir J, Samra Z, Ashkenazi S. Secondary bacteremia after rotavirus gastroenteritis in infancy. Pediatrics. 2006;117:224-6.

34. Niño DF, Sodhi CP, Hackam DJ. Necrotizing enterocolitis: new insights into pathogenesis and mechanisms. Nat Rev Gastroenterol Hepatol. 2016:13:590-600

35. Meropol SB, Stange KC, Jacobs MR, Weiss JK, Bajaksouzian S, Bonomo RA. Bacterial colonization and antibiotic resistance in a prospective cohort of newborn infants during the first year of life. Open Forum Infect Dis 2016; 3: ofw221.

36. Osthoff M, Gurtler N, Bassetti S, Balestra G, Marsch S, Pargger H, et al. Impact of MALDI-TOF-MS-based identification directly from positive blood cultures on patient management: a controlled clinical trial. Clin Microbiol Infect. 2017;23:78-85

37. Huang AM, Newton D, Kunapuli A, Gandhi TN, Washer LL, Lsip J, et al. Impact of rapid organism identification via matrix-assisted laser desorption/ ionization time-of-flight combined with antimicrobial stewardship team intervention in adult patients with bacteremia and candidemia. Clin Infect Dis. 2013:57:1237-45. 